\title{
Effectiveness of computerized ordering of test using unique hospital identification number to reduce pre analytical errors - a cross sectional study in a tertiary care hospital of south India
}

\author{
Jessy Sumangala Janardhanan ${ }^{1}$, Smitha Kalarikkal Satheesan ${ }^{2, *}$ \\ ${ }^{1}$ Associate Professor, Dept. of Biochemistry, Government Medical College, Thiruvananthapuram, Medical College, Kerala, \\ ${ }^{2}$ Assistant Professor, Dept. of Biochemistry, Government Medical College Thrissur, Kerala, India
}

*Corresponding Author:

Email: drsmithks@gmail.com

Received: $17^{\text {th }}$ March, 2018

Accepted: $30^{\text {th }}$ April, 2018

\begin{abstract}
Introduction: Laboratory test request forms are important communication device between the clinician and lab personnel. Studies have confirmed that most laboratory errors occur in the preanalytical phase clinicians are ordering investigations in paper request forms which are incomplete with regard to patient information and clinical details. Ordering the request through hospital computers using UHID number of the patient can reduce preanalytical errors.

Materials and Methods: Our study evaluated 806 Biochemistry lab report forms, by systematic sampling that reached Central Biochemistry lab from August to October 2016 and the same patients report forms retrieved from hospital computer using UHID number are compared for specific variables.

Results: Analysis of the study shows that information regarding sample collection time, sample receiving time in lab, reference range of the analyte, and name of the doctor are $100 \%$ entered in hospital computer but $0 \%$ entry in the paper request forms. General information of the patient $100 \%$ entry in hospital computer, in paper report form it is $98 \%$. But clinical notes, drug history and diagnosis not mentioned in both the hospital computer and the paper request forms.
\end{abstract}

Conclusion: Computerized entry and ordering of investigations by health care persons will reduce preanalytical errors.

Keywords: Preanalytical errors, Computerised ordering, Biochemistry lab reports, UHID Number.

\section{Introduction}

Quality assessment programs in laboratories mainly aims on analytical aspects in getting valid and accurate lab results. The process of laboratory medicine is typically divided into three main phases (preanalytical, analytical and post-analytical), with each of them variably affected by uncertainties and errors. ${ }^{1}$ Various studies have confirmed that errors in lab occur principally in the preanalytical phase affecting patient result and expenditure. Pre analytical phase should be subdivided into pre-pre analytical phase and preanalytical phase. Pre-pre analytical phase includes test request, patient or sample identification, sample collection, handling and transport, whereas pre analytical phase involves the steps of sample preparation for analysis such as centrifugation, aliquoting and sorting. It has been demonstrated that most errors occur in the pre-pre analytical phase by healthcare personnel who do not come under the direct control of the laboratory, where as pre analytical phase starts following the acceptance of specimen by the laboratory staff. 2,3

As laboratories play a crucial role in the patient diagnosis, incomplete data in the request form might significantly affect the lab service which in turn affects the patient's health care system. ${ }^{4,5}$ In the sample pathway, entering the patient information in the lab request form is always a bottle neck in sample processing. Issues such as illegible hand writing can cause non identification of patient. $^{6}$ Computerized ordering of test can reduce pre-analytical errors. In India very few studies were done to evaluate the data available in the patient request forms regarding pre analytical errors. This study is undertaken to evaluate the adequacy of computerized ordering of investigations using unique hospital identification (UHID) number in reducing pre- analytical errors.

\section{Aim}

1. To review the impact of unique hospital identification number in reducing pre- analytical errors.

2. To identify the incompleteness of data in printed Biochemistry laboratory request forms.

\section{Materials and Methods}

This study is conducted in Central Biochemistry lab of Government Medical College Thrissur, a 1400 bedded tertiary health care centre in Kerala. UHID number is assigned to each patient coming to this hospital from March 2015 onwards. General information regarding the patient such as name, age sex, income, address, OP/IP number, UHID number and date are entered by heath care personnel in the hospital computer which can there after retrieved from any hospital computer network by typing UHID number. During the period of study, the end to end system based management of patient and laboratory 
record keeping has been in migration phase. The study was done comparing the data in paper requisition form with data in lab report retrieved from system using UHID number, by collecting 806 biochemistry request forms by systematic sampling and the same patients lab reports retrieved from hospital computer using UHID number received at central Biochemistry lab over a period of 3 months from August to October 2016 are analyzed for the variables such as the patients name, age, gender, OP or IP, referring department, time of collection of sample, sample details, ordering of test, clinical details, probable diagnosis, and the name of requesting doctor have been verified. The information thus obtained is recorded in Microsoft Excel spread sheet and evaluated for statistical analysis. The lab receives around 900 samples per day. Patient's confidentiality has been maintained, name and hospital number will not be captured on the data sheet for analysis.

\section{Sample size}

$\mathrm{n}=\left\{\frac{\mathrm{Z}_{1-\alpha / 2} \sqrt{2 \mathrm{P} 0(1-\mathrm{P} 0)+\mathrm{Z} 1}-\beta \sqrt{\mathrm{Pa}(1-\mathrm{pa})}}{(\mathrm{Pa}-\mathrm{P} 0)^{2}}\right\}^{2}$

$\mathrm{P}_{0}=$ Population proportion (printed requisition form)

$\mathrm{Pa}=$ sample proportion (UHID results)

$\alpha=$ Significance level

$1-\beta=$ Power
From literature, the information coverage was about $50 \%$ and in the UHID results it was about more than $60 \%$.

\begin{tabular}{|l|c|}
\hline \multicolumn{1}{|c|}{$\begin{array}{c}\text { Hypothesis Testing for Single } \\
\text { Proportion }\end{array}$} & \\
\hline Population Proportion & 0.5 \\
\hline Sample Proportion & 0.6 \\
\hline Power (1- beta) \% & 90 \\
\hline Alpha error (\%) & 5 \\
\hline 1 or 2 sided & 2 \\
\hline Required sample size & 259 \\
\hline
\end{tabular}

The sampling method adopted is systematic random sampling and considering the design effect the sample size is 520 .Anticipating $10 \%$ dropout rate, final sample size is 572 .

Analysis: The difference in proportion between data collected using patients UHID number and printed Biochemistry request forms will be done using McNemar test. The incompleteness of printed biochemistry request forms will be expressed in percentage and 95\% confidence interval will be computed.

\section{Results}

A total of 806 patient's Biochemistry lab report forms and their lab data available from computer retrieved using UHID where compared are shown in table 1.

Table 1: Absent parameters in the lab request form and in hospital computer $(n=806)$

\begin{tabular}{|l|c|c|}
\hline \multicolumn{1}{|c|}{ Parameters } & Paper request form & Hospital computer \\
\hline Name & $0.10 \%$ & $0 \%$ \\
\hline Age & $0.50 \%$ & $0 \%$ \\
\hline Sex & $3.50 \%$ & $0 \%$ \\
\hline $\begin{array}{l}\text { Referral Department/Location of } \\
\text { Patient }\end{array}$ & $8.7 \%$ & $0 \%$ \\
\hline Date & $1.60 \%$ & $0 \%$ \\
\hline $\begin{array}{l}\text { Sample } \\
\text { Collection } \\
\text { Time }\end{array}$ & $100 \%$ & $0 \%$ \\
\hline $\begin{array}{l}\text { Sample } \\
\text { Receiving } \\
\text { Time }\end{array}$ & $100 \%$ & $0 \%$ \\
\hline Diagnosis & $100 \%$ & $100 \%$ \\
\hline $\begin{array}{l}\text { Name of } \\
\text { Clinician }\end{array}$ & $100 \%$ & $0 \%$ \\
\hline $\begin{array}{l}\text { Reference } \\
\text { range }\end{array}$ & $100 \%$ & $100 \%$ \\
\hline Drug history & & $0 \%$ \\
\hline
\end{tabular}

General Information: Regarding patient information name, age, sex unit, date are absent in the order $0.1 \%, 0.5 \%, 3.5 \%, 0 \%$ and $1.6 \%$ in the paper request forms whereas entry is $100 \%$ in the hospital computer.
Sample Information: Sample collection time, sample receiving time and reference range of the analyte are $100 \%$ entered in the hospital computer but the above information are not entered in the paper request form. 
Clinical Information: Diagnosis and drug history of the patient are not given in the paper request form and hospital computer. Name of the doctor is $100 \%$ entered in the hospital computer but no entry made in the paper request form.

\section{Discussion}

In India very few studies were done to assess pre analytical errors. Since $70 \%$ of medical diagnosis is influenced by medical lab reports, reduction of lab errors is of utmost importance. We evaluated manually written biochemistry lab reports and information in the lab reports traced from computer using UHID number. Our analysis shows that sample collection time, sample receiving time in the lab are not entered in the paper request forms, whereas $100 \%$ entry are made in the computer. Sample collection time is important in therapeutic drug monitoring. Also sample receiving time in lab is to be recorded. Delay in analysis can result in falsely low results could be recorded for arterial blood gas and bilirubin. ${ }^{7}$

Information regarding reference range are $100 \%$ obtained from the lab reports traced using UHID number whereas no entry are made in the lab report forms. Nearly $80 \%$ of physicians' medical decisions are based on information provided by laboratory reports. A test result by itself is of little value unless if it is reported with the inappropriate information for its interpretation. ${ }^{8}$

Provisional diagnosis is $100 \%$ traced from the lab report of hospital computer using UHID number but $0 \%$ entry are made in the paper lab report forms. Lab medical officer can interpret test results if provisional diagnosis is mentioned and can dispatch the test results without unnecessary delay when abnormal values are reported. This is consistent in the studies done by Sivakumar et al, ${ }^{5}$ Nutt et al, ${ }^{7}$ Nakleh et al, ${ }^{9}$ Kapil Bhatia et al ${ }^{10}$ Karunanandham et al. ${ }^{11}$

In the paper request form $91.3 \%$ has correctly mentioned location of the patient regarding referral department whereas the information was $100 \%$ obtained from the lab computer using UHID number. The absence of information regarding location of patient delay reporting critical values to the concerned clinicians as mentioned in the studies of Burton JL et al. ${ }^{12}$

The name of the doctor who requested the investigation can be traced $100 \%$ from the computer using UHID number but was not mentioned in the paper report forms. In the studies done Kapil Bhatia et $\mathrm{al}^{10}$ by Adegoke O A et al ${ }^{13}$ which shows that in only $4.3 \%$ cases doctor's name was not written on the requisition form as compared to the study by Khoury et $\mathrm{al}^{14}$ which showed that doctor's name cannot be identified in $17 \%$ of the request forms. Alarming results can be informed directly to the clinician by the lab physician if proper name of the clinician was there in the request form.
Drug history is not mentioned in the paper request form as well as can't be traced using UHID number. Many drugs can affect the interpretation of lab results and may even interfere with the assays. ${ }^{15,16}$

General information of the patient like Name, Age, Sex $99 \%$ entry made in the lab request form whereas $100 \%$ in the lab computer which agrees with the studies of Burton et al ${ }^{12}$ and Sivakumar et al. ${ }^{5}$

From the study we can conclude that among the preanalytical error patient/individual related error is more occurring during entering the information needed for the lab in the lab request form. This can be minimized by computerized ordering of the test by health professionals. When the sample reach the lab counter on entering the UIHD number a bar code is generated which is the sample label, it is pasted on the sample tube and this help to reduce misidentification of sample

Limitations of the Study: In the present study preanalytical variables like whether appropriate sample collection tube used, how long tourniquet was applied, how it was centrifuged, whether sample was lysed, sample were clotted mislabeling of sample, missing of sample were not studied. Only patient /individual related preanalytical errors were studied.

\section{Conclusion}

Proper laboratory request form has to be designed giving space for clinical notes, provisional diagnosis, and reference range of analytes have to be printed, ample space should be given for the clinician for stamping their seal, till the end to end software based system migration is in place. Drug history and clinical notes should be available in the paper request forms also when traced using UHID number. This can be achieved by conducting frequent training programmes and education programs to clinicians and paramedical staff to highlight the importance of filling relevant data in the request forms and also in the hospital computer.

\section{References}

1. Sharma P (2009) Pre-analytical variables and laboratory performance. Indian J Clin Biochem. April 2009;24(2):109-110.

2. Plebani M. Errors in clinical laboratories or errors in laboratory medicine? Clin Chem Lab Med. 2006;44(6):750-9. http:// dx.doi.org/10.1515/CCLM.2006.123.

3. Plebani M. Quality indicators to detect pre-analytical errors in laboratory testing. Clin Biochem Rev. Aug. 2012;33(3):85-8.

4. Lippi G, Guidi GC. Preanalytic indicators of laboratory performances and quality improvement of laboratory testing. Clin Lab. 2006;52(9-10):457-62.

5. Sivakumar et al (2015). A study of adequacy of completion of clinical biochemistry request forms, International Journal of Medical Research and Review. December,2015/Vol 3/Issue 11;1378-1382. 
6. Turner HE, Deans KA, Kite A, Croal BL. The effect of electronic ordering on pre-analytical errors in primary care. Ann Clin Biochem. Sep. 2013;50:485-488. doi: $10.1177 / 0004563213494184$

7. Nutt L, Zemlin AE, Erasmus RT. Incomplete laboratory request forms: the extent and impact on critical results at a tertiary hospital in South Africa. Ann Clin Biochem. 2008;45:463-466. doi: 10.1258/acb.2008.007252.

8. Yadav D (2015) Reference Interval for Clinical Laboratory Test Parameters. Biochem Anal Biochem 4:e158. doi:10.4172/2161-1009.1000e158.) 7. Nakhleh RE, Zarbo RJ. Surgical pathology specimen identification and accessioning: A college of American Pathologists QProbes Study of 1004115 cases from 417 institutions. Arch Pathol Lab Med. 1996;120:227-33.

9. Nakhleh RE, Zarbo RJ. Surgical pathology specimen identification and accessioning: A college of American Pathologists Q-Probes Study of 1004115 cases from 417 institutions. Arch Pathol Lab Med. 1996;120:227-33.

10. Kapil Bhatia, Pallavi Bhatia, Manasvi Praveen Kumar. Assessment of biochemistry laboratory requisition forms as a contributory factor to preanalytical errors in a tertiary care teaching hospital. International Journal of Contemporary Medical Research 2017;4(1):84-89.

11. Karunanandham S, Rajappa T, Jesudoss S, N Naveethalakshmi. A study of adequacy of completion of clinical biochemistry laboratory request forms. Int $\mathrm{J} \mathrm{Med}$ Res Rev. 2015;3(11):1378-1382.
12. Burton JL, Stephenson TJAre clinicians failing to supply adequate information when requesting a histopathological investigation? Journal of Clinical Pathology. 2001;54:806-808.

13. Adegoke O A, Idowu A A, Jeje O A. Incomplete laboratory request forms as a contributory factor to preanalytical errors in a Nigerian teaching hospital. Afr $J$ Biochem Res. 2011;5:82-85.

14. Khoury M, Burnett L, Mackay M. Error rates in Australian chemical pathology laboratories. MJA. 1996;165:128-130.

15. Young DS, Thomas DW, Friedman RB, Pestaner LC. Effects of drugs on clinical laboratory tests. Clin Chem. 1972;18:1041-303.

16. Surks ML, Sievert R. Drugs and thyroid function. $N$ Engl J Med. 1995;333:1688-94.

How to cite this article: Janardhanan JS, Satheesan SK. Effectiveness of computerized ordering of test using unique hospital identification number to reduce pre analytical errors - a cross sectional study in a tertiary care hospital of south India. Int $\mathrm{J}$ Clin Biochem Res. 2018;5(3):483-486. 